\title{
Successful management of chronic disseminated candidiasis in hematologic patients treated with high-dose liposomal amphotericin B: a retrospective study of the SEIFEM registry
}

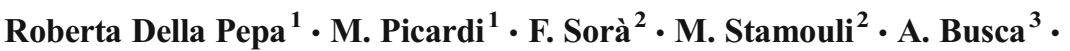

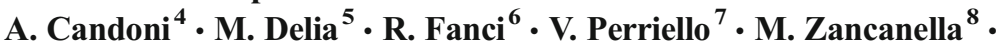

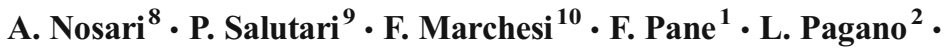 \\ on behalf of the SEIFEM group (Sorveglianza Epidemiologica Infezioni Fungine in Ematologia)
}

Received: 21 January 2016/Accepted: 3 April 2016/Published online: 14 April 2016

(C) The Author(s) 2016. This article is published with open access at Springerlink.com

\begin{abstract}
Purpose Chronic disseminated candidiasis (CDC) is a complication of Candida infection in immunocompromised patients, involving the liver and spleen, and rarely other organs. The aim of the study is to identify the best antifungal drug for hematologic immunocompromised patients with CDC.

Methods In this multicentric retrospective study, the charts of 20 patients with $\mathrm{CDC}$ following cytotoxic agent protocols for hematological malignancies, diagnosed from 2003 to 2013, were analyzed. The response to systemic antifungal therapy within 90 days from CDC diagnosis and the possible delay in chemotherapy plan, due to the infection, were evaluated.

Results Six patients were treated with high-dose (HD; $5 \mathrm{mg} / \mathrm{kg} /$ daily) liposomal amphotericin B (L-AmB), whereas three received standard-dose (SD) L-AmB (3 mg/kg/daily).
\end{abstract}

Roberta Della Pepa

roberta.dellapepa@unina.it

1 Department of Clinical Medicine and Surgery, Hematology, Federico II University, via Sergio Pansini, 5, Naples 80131, Italy

2 Hematology Catholic University Sacro Cuore, Rome, Italy

Hematology Le Molinette Hospital, Torino, Italy

4 Hematology University of Udine, Udine, Italy

5 Hematology University of Bari, Bari, Italy

6 Hematology University of Firenze, Florence, Italy

7 Hematology University of Perugia, Perugia, Italy

8 Hematology Niguarda Hospital, Milan, Italy

9 Hematology Pescara Hospital, Pescara, Italy

10 Haematology, Istituti Fisioterapici Ospitalieri (IFO), Rome, Italy
Azoles were given to six patients; the remaining five were treated with echinocandins. All patients treated with HD LAmB (6/6-100 \%) achieved complete resolution of CDC; one of them had to interrupt the chemotherapy program for the infection. In the SD L-AmB group, treatment failed in the $100 \%$ of cases and one patient had to delay chemotherapy for the infection. Of the six patients who received azoles, two achieved complete resolution of the infection, four experienced treatment failure, and only three performed chemotherapy as planned. Echinocandins treatment resulted in complete resolution of the infection in $2 / 5$ cases, partial response in $2 / 5$ cases, and failure in one case. In this group, $3 / 5$ patients completed chemotherapy as planned.

Conclusions This study shows that HD L-AmB was particularly effective against $\mathrm{CDC}$ in hematologic patients, allowing most patients to continue cytotoxic agent program.

Keywords Chronic disseminated candidiasis $\cdot$ Leukemia . Liposomal amphotericin B

\section{Introduction}

Invasive fungal infections (IFIs) are among the most relevant causes of morbidity and mortality for patients with hematological malignancies. The epidemiology of IFI has changed significantly over the last three decades; in the past, Candida spp. infection, mainly candidemias $[1,2]$, represented the most frequent IFI observed in these patients, but in recent years, invasive aspergillosis has become the most relevant IFI observed [3, 4]. Despite the reduced incidence, candidemia is characterized by a high mortality rate, as particularly demonstrated in various clinical and postmortem 
studies $[5,6]$. Chronic disseminated candidiasis (CDC) is the expression of a previous bloodstream Candida infection frequently misdiagnosed for the late onset of clinical and radiological manifestations. While in the past, it was frequently occurred [7,8], at present, this kind of complication is much more rare $[3,9]$. The most relevant consequence of this epidemiological trend is the lack of high-quality clinical trials assessing the best therapeutic approach for CDC. Another problem of this fungal complication is the delay in the treatment of the underlying hematological malignancy, with dramatic consequences (i.e., relapse) $[5,10]$.

In this multicentric study, we reviewed the clinical features of patients with hematological malignancies who developed CDC, identified according to the widely accepted diagnostic criteria [11]. First, we evaluated the best antifungal therapy on the basis of response rates assessed at 90 days after infection onset and then we considered the possible delay in chemotherapy plan due to $\mathrm{CDC}$, with consequences on overall survival (OS).

\section{Patients and methods}

A retrospective registry investigation was conducted in 35 Italian Hematology Tertiary Care Centres or University Hospitals to collect all proven/probable cases of CDC diagnosed from January 2003 to December 2013 [11]. Registered data were managed in accordance with the Italian Data Protection (Privacy) law. The survey was approved by the Ethics Committee of each participating site. All procedures followed were in accordance with the ethical standards of the responsible committee on human experimentation (institutional and national) and with the Helsinki Declaration of 1975, as revised in 2008 .

The supportive care and diagnostic work-up for febrile neutropenia were based on common measures to all SEIFEM (Sorveglianza Epidemiologica Infezioni Fungine in Ematologia) group centers. Patients with febrile neutropenia underwent at fever onset blood cultures from peripheral vein and central access, monitoring of serum galactomannan (biweekly), and chest X-rays and received broad-spectrum antibiotic therapy with beta-lactam and aminoglycoside agents. After 4 days of febrile neutropenia unresponsive to broad-spectrum antibiotics, a chest computed tomography (CT) scan was done and treatment with an intravenous antifungal drug started. Abdominal ultrasonography (US) or CT was performed if clinically indicated. $\beta$-D-glucan assay was routinely performed in only three centers involved in the study.

Only infections classified as "proven" or "probable" were included in this analysis. To improve diagnosis quality, the 2008 European Organization for Research and Treatment of Cancer/Mycoses Study Group (EORTC/MSG) definitions were used. To define proven CDC, the demonstration of fungal elements in diseased tissue was requested. To define a probable $\mathrm{CDC}$, clinical criteria were required, such as the imaging detection of nodule(s) with typical bull's eye aspect in the liver or spleen, as well as mycological finding in particular, an episode of candidemia in the previous 2 weeks [11].

For each case of CDC identified, a questionnaire eliciting the following data was requested: gender, age, kind of hematological malignancy, stage of the underlying malignancy and its treatment (i.e., induction or consolidation or salvage protocol, and autologous or allogenic hematopoietic stem cell transplant-HSCT), type of antifungal prophylaxis and duration, neutrophil count at $\mathrm{CDC}$ diagnosis, sites of $\mathrm{CDC}$ infection, diagnostic evaluation including microbiology (i.e., direct microscopy, cultures, and serology), imaging (CT and/or US), histology, specific diagnostic category of infection (proven/ probable), type of antifungal therapy (daily dose and duration) and response to various antifungal schedules, and 90-day OS.

The response to antifungal therapy within 90 days from $\mathrm{CDC}$ diagnosis was classified as complete response (CR; the disappearance of all signs and symptoms of infection and normalization of liver function tests, with a reduction or disappearance of all known lesions, as shown by CT and/or US abdominal scans, and no development of new lesion), partial response [PR; an improvement of all signs and symptoms of the disease, with a reduction of at least $25 \%$ of the diameter of the lesion(s)], stable response (SR; only a slight improvement of all symptoms and signs of the disease, with reduced lesion diameter from 0 to $25 \%$ ), and failure (F; persistence of Candida spp. in blood samples or specimens from other sites, in association with worsening symptoms or clinical signs of the disease, new sites of the disease, or worsening of preexisting injuries) [12]. Candida attributable mortality was considered death due to fungal infection.

The OS included all-cause mortality at 90-day follow-up from CDC diagnosis and was generated using the KaplanMeier product limit estimator [13, 14].

\section{Results}

Twenty-six of the 35 centers participating in the survey did not observe any cases suspected for CDC during the study period. A total of 25 cases with suspected CDC in only nine centers were identified. Five patients were excluded due to the lack of complete documentation for the diagnosis of CDC, despite the presence of suggestive signs and symptoms (Fig. 1). The clinical characteristics of the 20 evaluable patients (M/F 12/8; median age 50 years, range 19-62 years) who developed CDC identified according to standardized diagnostic criteria are detailed in Table 1. The majority of patients had acute leukemia. The infection was preceded in all patients by intensive chemotherapy protocols; $13 / 20(65 \%)$ received 
Fig. 1 Study flow chart. *According to the 2008 EORTC/ MSG criteria [11]

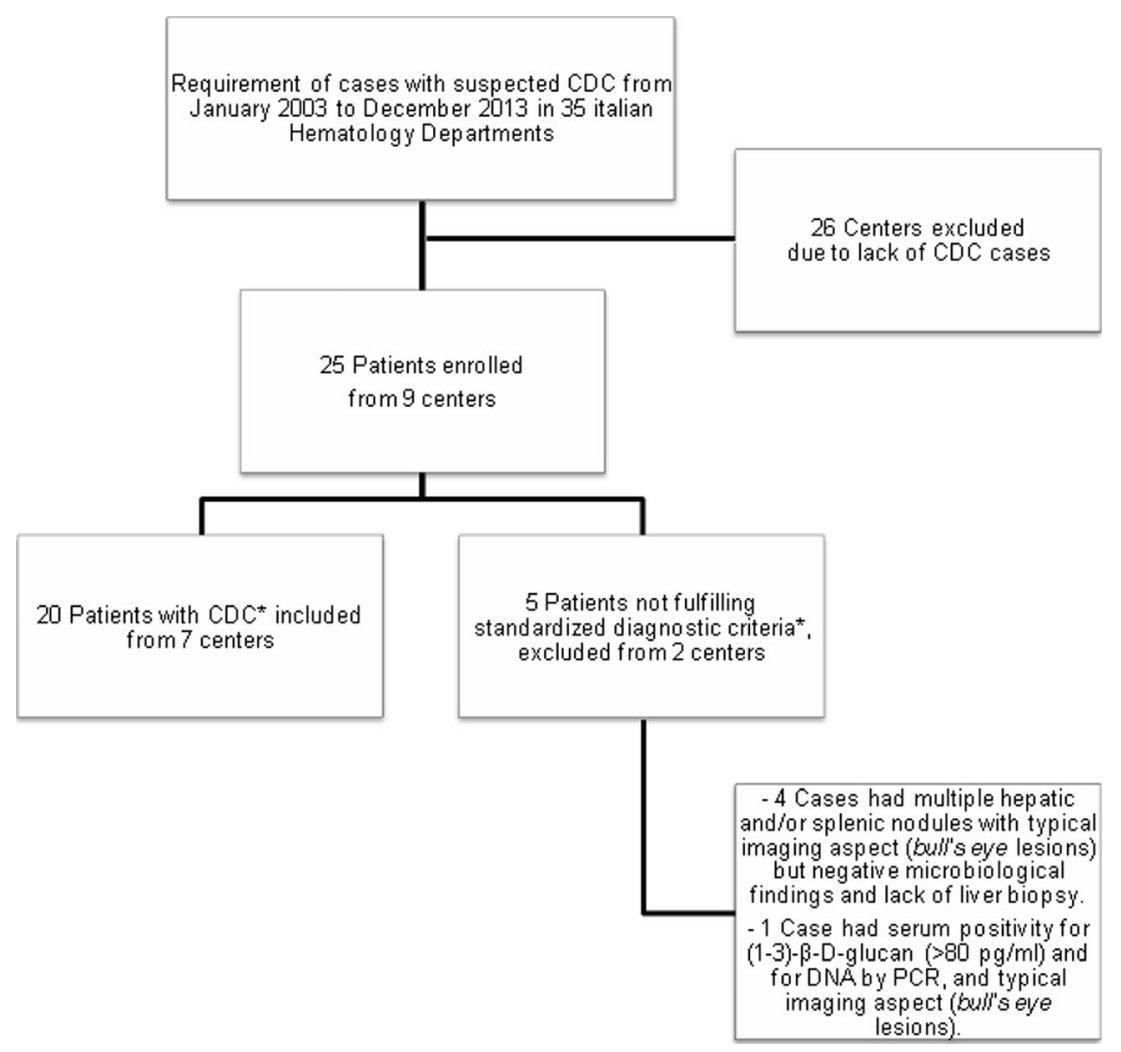

cytarabine-based regimens and three (15\%) HSCTs (allogenic, two cases; autologous, one case). Before the infection, 14/ $20(70 \%)$ had severe neutropenia (neutrophil count $<500 / \mu \mathrm{l}$ ) for a median time of 14 days (range 2-30 days). Antifungal prophylaxis was done in 14/20 patients: $65 \%$ of them had azoles in oral formulation (six fluconazole; six itraconazole; one voriconazole) and 1 patient received topic prophylaxis with nystatin. No prophylaxis was given to $6 / 20$ patients (30\%).

The most common clinical manifestation of CDC (19/20 patients - $95 \%$ ) was persistent fever unresponsive to conventional antibiotics. Abdominal pain was the second most common finding following fever (15/20 patients - $75 \%)$. Other signs and symptoms included diarrhea (8/20-40\%), jaundice $(6 / 20-30 \%)$, vomiting $(2 / 20-10 \%)$, dyspnea $(2 / 20-10 \%)$, and ileotyphlitis $(2 / 20-10 \%)$.

Trend in the distribution of Candida species in our 20 patients is shown in Table 1. The majority of infections were due to Candida non-albicans strains. There were 13 cases with diagnosis of probable CDC and seven cases with diagnosis of proven CDC.

All patients were treated for CDC infection by systemic antifungal agents. Liposomal amphotericin B (LAmB) was given to $9 / 20$ (45\%) patients; of them, six received high-dose (HD) L-AmB (5 $\mathrm{mg} / \mathrm{kg} /$ day) and three standard-dose (SD) L-AmB (3 $\mathrm{mg} / \mathrm{kg} /$ day). Azoles were used in 6/20 (30 \%) patients (fluconazole
$400 \mathrm{mg} /$ day in $3 / 6$, voriconazole $4 \mathrm{mg} / \mathrm{kg}$ twice daily in $2 / 6$, itraconazole $400 \mathrm{mg} /$ day in 1/6). Finally, 5/20 (25\%) patients were treated with echinocandins (caspofungin $50 \mathrm{mg} /$ day in 4/5; anidulafungin $100 \mathrm{mg}$ / day in 1/5). All patients treated with HD L-AmB (6/6$100 \%$ ) achieved CR of CDC infection; of them, only one patient $(16 \%)$ interrupted the chemotherapy scheduled program for the infection. In the SD L-AmB group, there was $100 \%$ of F (one death; two SR) and $1 / 3(33 \%)$ patient delayed chemotherapy for the infection. Of the six patients receiving azoles, two (33\%) achieved $\mathrm{CR}$ of the infection, four $(66 \%)$ experienced F; only 3/6 (50 \%) patients performed chemotherapy treatment as planned. Echinocandins treatment resulted in CR of the infection in 2/5 (40\%) cases, PR in $2 / 5$ (40\%) cases, and $\mathrm{F}$ in one case $(20 \%)$. In this group, $3 / 5(60 \%)$ patients underwent chemotherapy as planned. The outcome of CDC according to the microbiological findings is reported in Table 1. The outcome of CDC according to the various antifungal schedules in the 20 patients treated is reported in Fig. 2. Overall, the median duration of antifungal therapy was 30 days (range 10-90 days).

Within 90 days from CDC diagnosis, the overall mortality rate was $60 \%$. In particular, 3/20 patients died because of infection, while 9/20 died for the underlying malignancy. Thus, Candida attributable mortality rate was $25 \%$. 


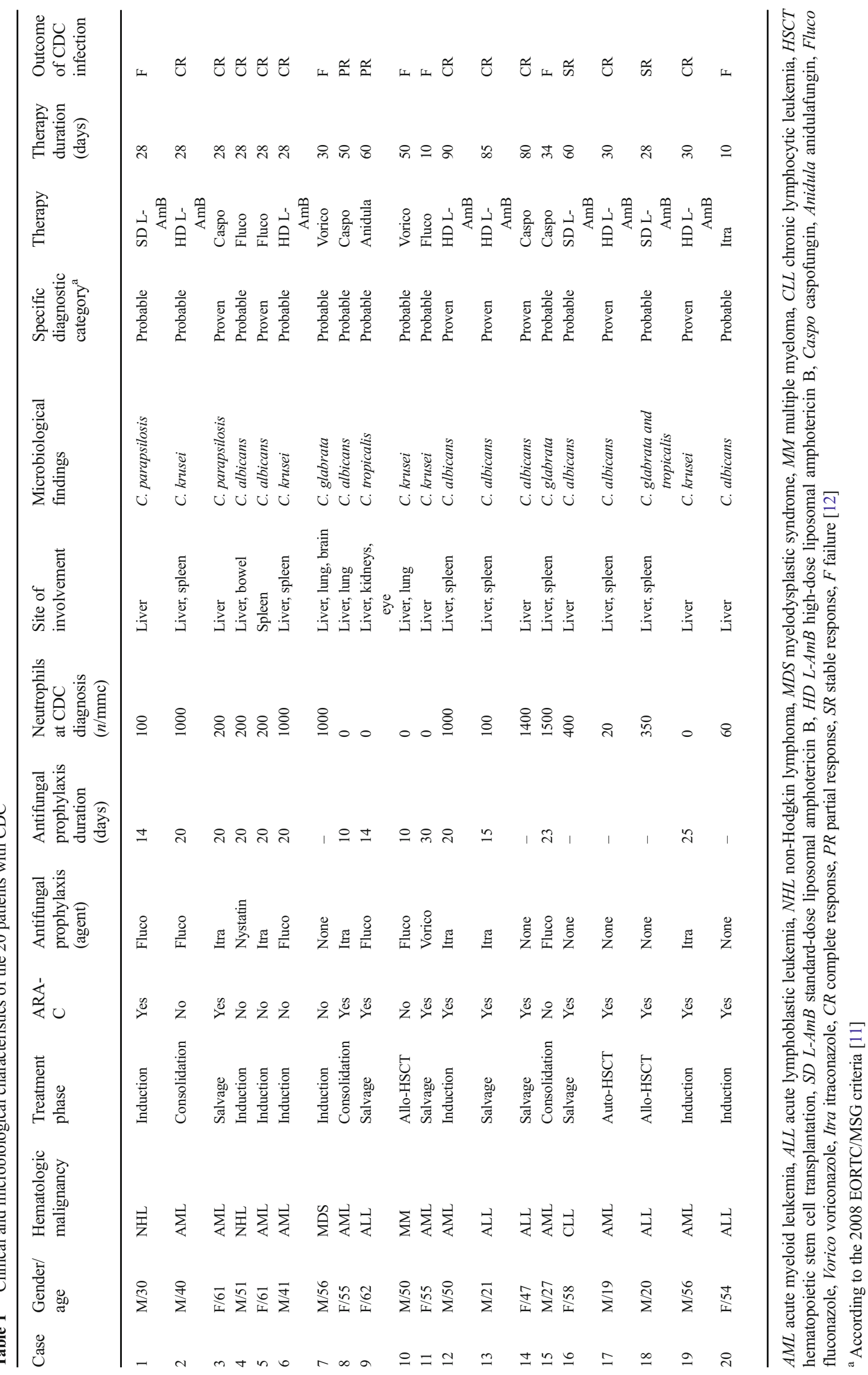




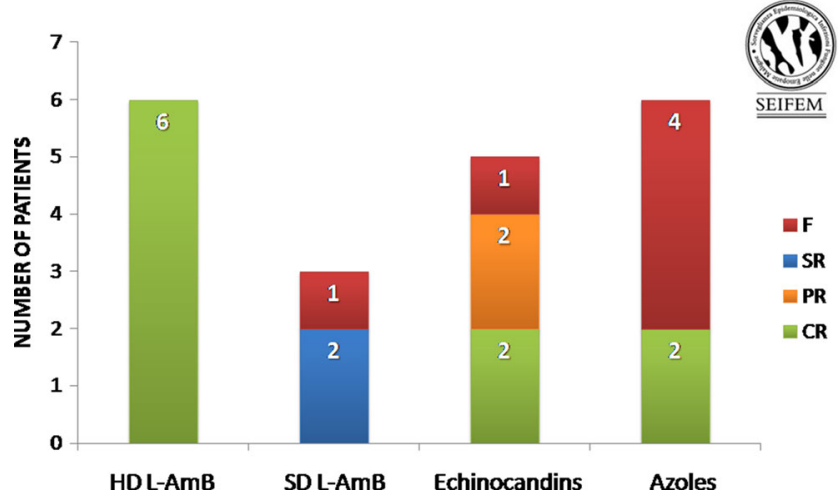

Fig. 2 Response to antifungal therapy in 20 patients with CDC. $S D L$ $A m B$ standard-dose liposomal amphotericin B, $H D L-A m B$ high-dose liposomal amphotericin $\mathrm{B}, C R$ complete response, $P R$ partial response, $S R$ stable response, $F$ failure [12]

The OS of patients who received HD L-AmB was better than those treated with other antifungals (66 vs. $21 \%$, respectively; $p=0.04$ ). It may be a consequence of the greater number of infectious complications obtaining $\mathrm{CR}$ due to HD L$\mathrm{AmB}$ then to the other antifungal schedules, and, thus, a consequence of the regular continuation of chemotherapy program for the underlying hematologic malignancy (Fig. 3).

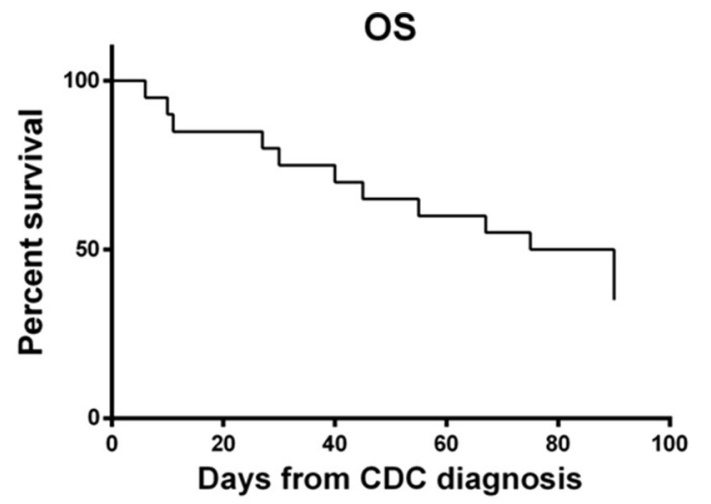

A

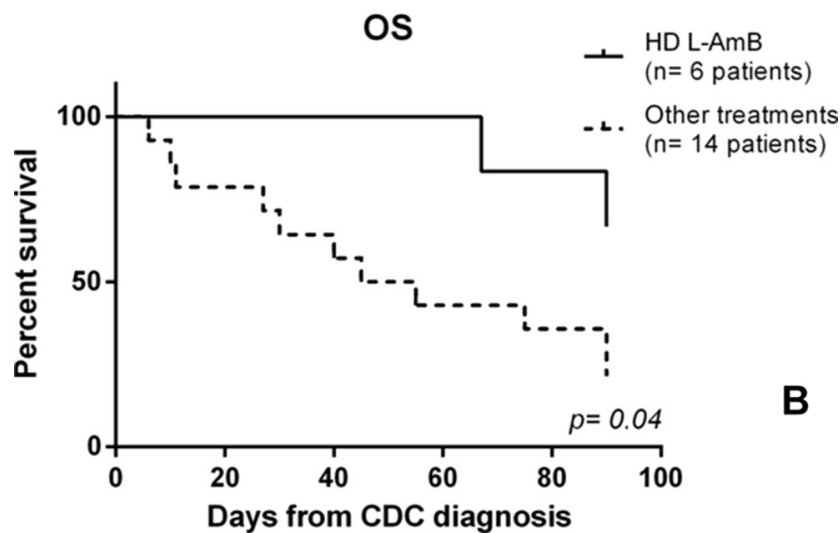

Fig. 3 a Overall survival (OS - $40 \%$ ) of all 20 patients with hematologic malignancy and chronic disseminated candidiasis (CDC) at 90 days from infection diagnosis. b OS of patients treated with high-dose (HD) liposomal amphotericin B (L-AmB) (66 \%) versus other antifungal treatments $(21 \%)$ at 90 days from infection diagnosis

\section{Discussion}

$\mathrm{CDC}$ is a rare complication representing a distinct form of disseminated Candida infection; it mainly involves the liver, spleen, and occasionally kidneys and other organs [15]. CDC mostly occurs after severe and prolonged neutropenia, which is more often seen in patients with hematological malignancies [3]. In the last few years, EORTC/MSG definitions have been modified applying more restrictive diagnostic criteria. 2008 EORTC/MSG criteria identify three different categories to define IFI: possible, probable, and proven [11]. Regarding $\mathrm{CDC}$, the most important consequence of this re-visitation is an improvement of the quality of diagnosis with marked reduction of the probable cases.

Only few retrospective series have been published in the last few years. In 2002, Pagano et al. evaluated a group of 29 patients with hematological malignancies who developed CDC. This retrospective study showed a low mortality rate and a high efficacy of L-AmB; only one patient died of $\mathrm{CDC}$, while 11 had to delay the treatment for their underlying malignancy because of the persistence of infection [16]. Another retrospective series, published by Ellis et al. in 2006, compared the efficacy and safety of caspofungin and L-AmB in treating hepatosplenic candidosis. This study showed that neither of their five CDC cases responded to caspofungin compared with $2 / 3$ cases successfully treated with L-AmB [17]. More recently, in 2012, De Castro et al. published a retrospective series of 24 hematologic patients with hepatosplenic candidiasis. Neutropenia duration of $>1$ month and hematological relapse were both strongly predictive factors of death, while delayed chemotherapy treatment because of hepatosplenic candidosis was not a mortality risk factor [18].

In the absence of high-quality clinical trials, $\mathrm{CDC}$ treatment as reported by the Scientific Society is, above all, based on small series and/or expert opinions [3, 15, 16, 18]. In the main international guidelines, there is a marked discrepancy between the recommendation for the treatment of CDC in immunocompetent and immunocompromised patients and also for the latter, not all guidelines are in agreement [19-22]. The guidelines of the Infectious Diseases Society of America suggest fluconazole $400 \mathrm{mg}(6 \mathrm{mg} / \mathrm{kg})$ daily for stable patients (recommendation grade: AIII), and L-AmB 3-5 mg/kg daily or amphotericin B deoxycholate $0.5-0.7 \mathrm{mg} / \mathrm{kg}$ daily for severely ill patients (AIII), continuing with fluconazole once the patient is stable (BIII) [19]. For the eradication of CDC, the guidelines of the European Society of Clinical Microbiology and Infection Diseases recommend an 8-week treatment with L-AmB (AIII) because of the potential accumulation in the reticulo-endothelial system [20], or fluconazole for $>3$ months or other azoles (BIII). This recommendation only considers non-neutropenic adult patients [21]. Recommendations of the Infectious Diseases Working Party of the German Society of 
Hematology and Oncology for hematologic patients with CDC indicate the use of fluconazole $(400-800 \mathrm{mg}$ /day) if the Candida strain is isolated and proven to be susceptible to this molecule (BIII), and echinocandins or L-AmB as initial therapy in unstable or refractory patients (BIII). Voriconazole is considered an alternative option (CIII) [22]. However, the appropriateness of an anti-infectious treatment in patients with $\mathrm{CDC}$, particularly in those patients with hematological disease, has a crucial role not only for reducing the mortality rates due to the infection but also for the marked impact on subsequent cytotoxic agent schedules for the underlying malignancy.

Even if there is a not homogeneous indication, all international guidelines agree that $\mathrm{L}-\mathrm{AmB}$ seems to be one of the better choice in the treatment of this complication. Considering the great difficulty in obtaining timely and effective responses that allow the respect of the chemotherapy plane, some authors have suggested to increase the dosage of L-AmB reaching $10 \mathrm{mg} / \mathrm{kg} / \mathrm{day}$ as in other fungal complications, but this schedule provided no additional benefit and higher rates of nephrotoxicity compared to the standard dose [23]. On the contrary, the use of L-AmB at $5 \mathrm{mg} / \mathrm{kg} / \mathrm{day}$ is characterized by a mild toxicity that did not differ from conventional dose $[24,25]$. Considering that in autopsy tissue, the highest concentrations of L-AmB were found in the liver and spleen, followed by the kidney, lung, myocardium, and brain tissue [26], it could be the correct dosage in this subset of patients.

Our study collected all cases of CDC developed in Italian Hematology Departments during a 10 -year period. Only 20 cases were collected, and in the majority of institutions, no proven/probable cases were observed. It confirms that this is a very rare complication. As a consequence, it is very difficult to draw-up studies able to answer questions about what is the best treatment. Our study has several limitations. It is a retrospective study which restricts the value of the results; hence, our findings need to be validated in a prospective multicenter trial. Moreover, this is a very heterogeneous series of cases with different characteristics (i.e., different underlying hematological malignancies, different hematological malignancy stages, different cytotoxic agent schedules, and different antifungal prophylaxis approaches).

The results of this retrospective study, with all the limits due to the small number of cases treated for each group, seem to confirm that higher doses of L-AmB are characterized by better efficacy than conventional doses or other antifungal agents (azoles and echinocandins), supporting the hypothesis that HD L-AmB acts as fungicide in the liver and/or spleen [26]. We observed CR of infection in $100 \%$ of patients treated with this regimen and a significantly better OS; above all, $84 \%$ of them were able to continue the chemotherapy program for the underlying hematologic malignancy. Additional studies are needed to assess the efficacy of highdose L-AmB against CDC in a larger hematologic patient population.

Compliance with ethical standards The survey was approved by the Ethics Committee of each participating site. All procedures followed were in accordance with the ethical standards of the responsible committee on human experimentation (institutional and national) and with the Helsinki Declaration of 1975, as revised in 2008.

Conflict of interest The authors declare that they have no competing interests.

Open Access This article is distributed under the terms of the Creative Commons Attribution-NonCommercial 4.0 International License (http:// creativecommons.org/licenses/by-nc/4.0/), which permits any noncommercial use, distribution, and reproduction in any medium, provided you give appropriate credit to the original author(s) and the source, provide a link to the Creative Commons license, and indicate if changes were made.

\section{References}

1. Beck-Sague C, Jarvis WR (1993) Secular trends in the epidemiology of nosocomial fungal infections in the United States, 1980 1990. National Nosocomial Infections Surveillance System. J Infect Dis 167(5):1247-1251

2. Jarvis WR, Martone WJ, Suppl A (1992) Predominant pathogens in hospital infections. J Antimicrob Chemother 29:19-24

3. Pagano L, Caira M, Candoni A, Offidani M, Fianchi L, Martino B, Pastore D, Picardi M, Bonini A, Chierichini A, Fanci R, Caramatti C, Invernizzi R, Mattei D, Mitra ME, Melillo L, Aversa F, Van Lint MT, Falcucci P, Valentini CG, Girmenia C, Nosari A (2006) The epidemiology of fungal infections in patients with hematologic malignancies: the SEIFEM-2004 study. Haematologica 91(8):10681075

4. Pagano L, Caira M, Rossi G, Tumbarello M, Fanci R, Garzia MG, Vianelli N, Filardi N, De Fabritiis P, Beltrame A, Musso M, Piccin A, Cuneo A, Cattaneo C, Aloisi T, Riva M, Rossi G, Salvadori U, Brugiatelli M, Sannicolo S, Morselli M, Bonini A, Viale P, Nosari A, Aversa F, Hema e-Chart Group I (2012) A prospective survey of febrile events in hematological malignancies. Ann Hematol 91(5): 767-774. doi:10.1007/s00277-011-1373-2

5. Lehrnbecher T, Frank C, Engels K, Kriener S, Groll AH, Schwabe D (2010) Trends in the postmortem epidemiology of invasive fungal infections at a university hospital. J Infect 61(3):259-265. doi: 10.1016/j.jinf.2010.06.018

6. Lewis RE, Cahyame-Zuniga L, Leventakos K, Chamilos G, BenAmi R, Tamboli P, Tarrand J, Bodey GP, Luna M, Kontoyiannis DP (2013) Epidemiology and sites of involvement of invasive fungal infections in patients with haematological malignancies: a 20-year autopsy study. Mycoses 56(6):638-645. doi:10.1111/myc.12081

7. Jarvis WR (1995) Epidemiology of nosocomial fungal infections, with emphasis on Candida species. Clin Infect Dis 20(6):15261530

8. Perduca M, Marangoni E, Guanziroli A, Romero E, Filice G (1995) Fungaemia in hospitalized patients. Mycoses 38(9-10):385-387

9. Nosari AM, Caira M, Pioltelli ML, Fanci R, Bonini A, Cattaneo C, Castagnola C, Capalbo SF, De Fabritiis P, Mettivier V, Morselli M, Pastore D, Aversa F, Rossi G, Pagano L, Hema e-Chart Group I 
(2013) Hema e-Chart registry of invasive fungal infections in haematological patients: improved outcome in recent years in mould infections. Clin Microbiol Infect 19(8):757-762. doi:10.1111/14690691.12014

10. Masood A, Sallah S (2005) Chronic disseminated candidiasis in patients with acute leukemia: emphasis on diagnostic definition and treatment. Leuk Res 29(5):493-501. doi:10.1016/j.leukres. 2004.10.003

11. De Pauw B, Walsh TJ, Donnelly JP, Stevens DA, Edwards JE, Calandra T, Pappas PG, Maertens J, Lortholary O, Kauffman CA, Denning DW, Patterson TF, Maschmeyer G, Bille J, Dismukes WE, Herbrecht R, Hope WW, Kibbler CC, Kullberg BJ, Marr KA, Munoz P, Odds FC, Perfect JR, Restrepo A, Ruhnke M, Segal BH, Sobel JD, Sorrell TC, Viscoli C, Wingard JR, Zaoutis T, Bennett JE, European Organization for R, Treatment of Cancer/ Invasive Fungal Infections Cooperative G, National Institute of A, Infectious Diseases Mycoses Study Group Consensus G (2008) Revised definitions of invasive fungal disease from the European Organization for Research and Treatment of Cancer/Invasive Fungal Infections Cooperative Group and the National Institute of Allergy and Infectious Diseases Mycoses Study Group (EORTC/ MSG) Consensus Group. Clin Infect Dis 46(12):1813-1821. doi: $10.1086 / 588660$

12. Segal BH, Herbrecht R, Stevens DA, Ostrosky-Zeichner L, Sobel J, Viscoli C, Walsh TJ, Maertens J, Patterson TF, Perfect JR, Dupont B, Wingard JR, Calandra T, Kauffman CA, Graybill JR, Baden LR, Pappas PG, Bennett JE, Kontoyiannis DP, Cordonnier C, Viviani MA, Bille J, Almyroudis NG, Wheat LJ, Graninger W, Bow EJ, Holland SM, Kullberg BJ, Dismukes WE, De Pauw BE (2008) Defining responses to therapy and study outcomes in clinical trials of invasive fungal diseases: Mycoses Study Group and European Organization for Research and Treatment of Cancer consensus criteria. Clin Infect Dis 47(5):674-683. doi:10.1086/590566

13. Kaplan ELM, P (1958) Nonparametric estimation from incomplete observations. J Amer Statist Assn 53:457-481

14. Kaplan EL (1983) This week's citation classic. Current Contents 24:14

15. Blade J, Lopez-Guillermo A, Rozman C, Granena A, Bruguera M, Bordas J, Cervantes F, Carreras E, Sierra J, Montserrat E (1992) Chronic systemic candidiasis in acute leukemia. Ann Hematol 64(5):240-244

16. Pagano L, Mele L, Fianchi L, Melillo L, Martino B, D'Antonio D, Tosti ME, Posteraro B, Sanguinetti M, Trape G, Equitani F, Carotenuto M, Leone G (2002) Chronic disseminated candidiasis in patients with hematologic malignancies. Clinical features and outcome of 29 episodes. Haematologica 87(5):535-541

17. Ellis M, Frampton C, Joseph J, Alizadeh H, Kristensen J, Hauggaard A, Shammas F (2006) An open study of the comparative efficacy and safety of caspofungin and liposomal amphotericin $\mathrm{B}$ in treating invasive fungal infections or febrile neutropenia in patients with haematological malignancy. J Med Microbiol 55(Pt 10):1357-1365. doi:10.1099/jmm.0.46452-0

18. De Castro N, Mazoyer E, Porcher R, Raffoux E, Suarez F, Ribaud P, Lortholary O, Molina JM (2012) Hepatosplenic candidiasis in the era of new antifungal drugs: a study in Paris 2000-2007. Clin Microbiol Infect 18(6):E185-187. doi:10.1111/j.1469-0691.2012. 03819.x

19. Pappas PG, Kauffman CA, Andes D, Benjamin DK Jr, Calandra TF, Edwards JE Jr, Filler SG, Fisher JF, Kullberg BJ, OstroskyZeichner L, Reboli AC, Rex JH, Walsh TJ, Sobel JD, Infectious Diseases Society of A (2009) Clinical practice guidelines for the management of candidiasis: 2009 update by the Infectious Diseases Society of America. Clin Infect Dis 48(5):503-535. doi:10.1086/ 596757

20. Chakraborty KK, Naik SR (2000) In situ liposomal preparation containing amphotericin B: related toxicity and tissue disposition studies. Pharm Dev Technol 5(4):543-553. doi:10.1081/PDT100102037

21. Cornely OA, Bassetti M, Calandra T, Garbino J, Kullberg BJ, Lortholary O, Meersseman W, Akova M, Arendrup MC, ArikanAkdagli S, Bille J, Castagnola E, Cuenca-Estrella M, Donnelly JP, Groll AH, Herbrecht R, Hope WW, Jensen HE, Lass-Florl C, Petrikkos G, Richardson MD, Roilides E, Verweij PE, Viscoli C, Ullmann AJ, Group EFIS (2012) ESCMID* guideline for the diagnosis and management of Candida diseases 2012: non-neutropenic adult patients. Clin Microbiol Infect 18(Suppl 7):19-37. doi:10. 1111/1469-0691.12039

22. Mousset S, Buchheidt D, Heinz W, Ruhnke M, Cornely OA, Egerer G, Kruger W, Link H, Neumann S, Ostermann H, Panse J, Penack O, Rieger C, Schmidt-Hieber M, Silling G, Sudhoff T, Ullmann AJ, Wolf HH, Maschmeyer G, Bohme A (2014) Treatment of invasive fungal infections in cancer patients-updated recommendations of the Infectious Diseases Working Party (AGIHO) of the German Society of Hematology and Oncology (DGHO). Ann Hematol 93(1):13-32. doi:10.1007/s00277-013-1867-1

23. Cornely OA, Maertens J, Bresnik M, Ebrahimi R, Ullmann AJ, Bouza E, Heussel CP, Lortholary O, Rieger C, Boehme A, Aoun M, Horst HA, Thiebaut A, Ruhnke M, Reichert D, Vianelli N, Krause SW, Olavarria E, Herbrecht R, AmBiLoad Trial Study G (2007) Liposomal amphotericin B as initial therapy for invasive mold infection: a randomized trial comparing a high-loading dose regimen with standard dosing (AmBiLoad trial). Clin Infect Dis 44(10):1289-1297. doi:10.1086/514341

24. Adler-Moore JP, Gangneux JP, Pappas PG (2016) Comparison between liposomal formulations of amphotericin B. Med Mycol. doi: 10.1093/mmy/myv111

25. Ellis M, Spence D, de Pauw B, Meunier F, Marinus A, Collette L, Sylvester R, Meis J, Boogaerts M, Selleslag D, Krcmery V, von Sinner W, MacDonald P, Doyen C, Vandercam B (1998) An EORTC international multicenter randomized trial (EORTC number 19923) comparing two dosages of liposomal amphotericin B for treatment of invasive aspergillosis. Clin Infect Dis 27(6):14061412

26. Vogelsinger H, Weiler S, Djanani A, Kountchev J, Bellmann-Weiler R, Wiedermann CJ, Bellmann R (2006) Amphotericin B tissue distribution in autopsy material after treatment with liposomal amphotericin B and amphotericin B colloidal dispersion. J Antimicrob Chemother 57(6):1153-1160. doi:10.1093/jac/dk1141 